\title{
APROXIMACIÓN A UNA ECONOMÍA POLIITICA DE LOS CONFLICTOS SOCIOAMBIENTALES EN MÉXICO: EXTRACTIVISMO, RECURSOS NATURALES ESTRATÉGICOS Y FRACTURAS METABÓLICAS
}

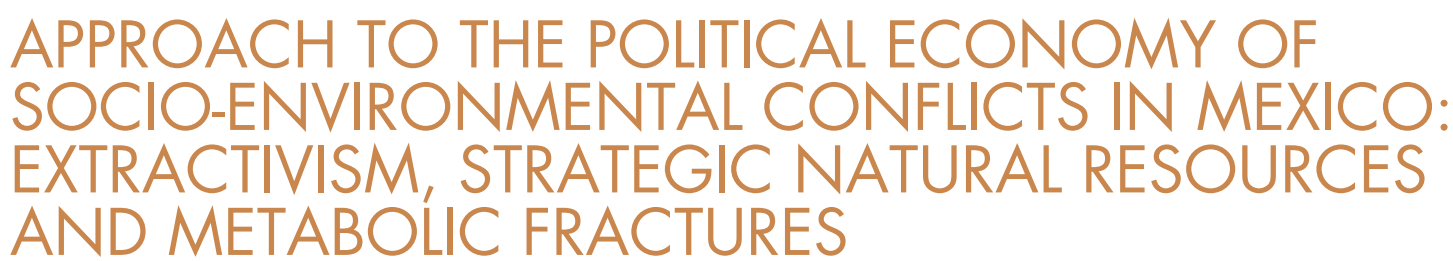

Josemanuel Luna-Nemecio*

RESUMEN: El estudio se basó en la perspectiva epistemológica y teórica de la crítica de la economía política para proponer un acercamiento a la conceptualización y caracterización de los conflictos socioambientales a partir de su especificidad histórica en torno al desarrollo del capitalismo contemporáneo. Para ilustrar lo que se define como una conflictividad socioambiental específicamente neoliberal, se presentaron datos sobre la extracción de los recursos naturales estratégicos que forman parte nodal del metabolismo social-natural en México, cuya tasa de explotación se establece como condición de posibilidad necesaria - más no suficiente- para la eventual emergencia de conflictos socioambientales. La investigación se hizo mediante un análisis cualitativo de documentos que permitió dar cuenta de los vacíos y contradicciones en torno a la conceptualización y al abordaje científico de los conflictos socioambientales en México en referencia al extractivismo, el metabolismo social y los recursos naturales estratégicos. Los resultados del estudio permitieron: 1) Definir la especificidad histórica de los conflictos socioambientales como un producto de la sobreexplotación creciente de la naturaleza, de sobreconsumo de materiales y energía, y de excreta inconmensurable de residuos sólidos y de alta toxicidad que caracterizan los procesos productivos y consuntivos en el neoliberalismo. 2) Bajo la lógica de la crítica de la economía política, la emergencia de conflictos socioambientales se caracteriza por la creciente de la devastación ambiental, la degradación del tejido social y la producción social del riesgo y vulnerabilidad múltiple de las condiciones práctico materiales y simbólicas identitarias de la vida de las comunidades agraviadas. 3) Se presentaron datos sobre la extracción de minerales, hidrocarburos, biomasa y agua, que ha tenido lugar en México a lo largo del neoliberalismo, en tanto que estos recursos representan recursos naturales estratégicos para el desarrollo del capital nacional y transnacional; esta información se relaciona con la configuración de conflictos socioambien-

Recibido: 23.11.19 Aceptado: 17.12.20

\footnotetext{
* El presente trabajo forma parte de los resultados de investigación del proyecto "Conflictos socioambientales de corte hídrico en el estado de Morelos: un análisis territorial sobre la defensa del agua ante el desarrollo urbano-industrial impulsado por el Proyecto Integral Morelos", financiado por el Consejo Nacional de Ciencia y Tecnología de México (CONACYT), en el marco del Programa de Estancias Posdoctorales por México 2020.
}

Doctor en Geografía por la Universidad Nacional Autónoma de México, Posdoctorante en la Unidad Académica en Estudios del Desarrollo de la Universidad Autónoma de Zacatecas. Miembro del Sistema Nacional de Investigadores del CONACYT. Sus líneas de investigación son la crítica de la economía política con énfasis en estudios territoriales, recursos naturales estratégicos, cambio climático, socioformación y desarrollo social sostenible. Correo: josmaluna2@gmail.com. ORCID: https://orcid.org/0000-0002-6850-3443 
tales y la fractura metabólica de los límites ecológicos planetarios que implica. El estudio concluye que los conflictos socioambientales son producto del desarrollo de tecnología capitalista nociva y fuerzas productivas ambientalmente destructivas y degradantes de tejido social que emergen y se consolidan territorialmente bajo un contexto neoliberal. Por lo que la conflictividad socioambiental puede ser vista como una forma problemática y contradictoria de las relaciones sociales basadas en escenarios de pugna y confrontación referentes a la apropiación, saqueo y contaminación de los territorios que ponen en riesgo las condiciones para la reproducción de la naturaleza y la sociedad.

Palabras clave: conflictos socioambientales; devastación ambiental; límites ecológicos planetarios; metabolismo social-natural; recursos naturales estratégicos.

ABSTRACT: The study was based on the epistemological and theoretical perspective of critique of political economy to propose an approach to the conceptualization and characterization of socio-environmental conflicts based on their historical specificity in the development of contemporary capitalism. To illustrate what is defined as a specifically neoliberal socio-environmental conflict, data were presented on the extraction of strategic natural resources that form a nodal part of the social-natural metabolism in Mexico, whose rate of exploitation is established as a necessary-but not sufficient- condition of possibility for the eventual emergence of socio-environmental conflicts. The research was carried out through a qualitative analysis of documents that made it possible to account for the gaps and contradictions around the conceptualization and scientific approach to socio-environmental conflicts in Mexico in reference to extractivism, social metabolism, and strategic natural resources. The results of the study allowed: 1) to define the historical specificity of socio-environmental conflicts as a product of the growing overexploitation of nature, overconsumption of materials and energy, and immeasurable excretion of solid waste and high toxicity that characterizes the productive and consumptive processes in neoliberalism. 2) Under the logic of the critique of political economy, the emergence of socio-environmental conflicts is characterized by the increasing environmental devastation, the degradation of the social fabric and the social production of risk, and multiple vulnerabilities of the practical material and symbolic identity conditions of the life of the aggrieved communities. 3) Data was presented on the extraction of minerals, hydrocarbons, biomass, and water, which has taken place in Mexico throughout neoliberalism, as these resources represent strategic natural resources for the development of national and transnational capital; this information is related to the configuration of socio-environmental conflicts and the metabolic fracture of the planetary ecological limits that said extractivism activities imply. The study concludes that socio-environmental conflicts are the product of the development of harmful capitalist technology and environmentally destructive and degrading productive forces that degrade the social fabric and emerge and consolidate territorially under a neoliberal context. Thus, socio-environmental conflict can be seen as a problematic and contradictory form of social relations based on scenarios of conflict and confrontation regarding the appropriation, plundering and contamination of territories that jeopardize the conditions for the reproduction of nature and society.

Key words: environmental devastation; planetary ecological limits; social-natural metabolism; socio-environmental conflicts; strategic natural resources. 


\section{INTRODUCCIÓN}

A la hora de lanzar una mirada panorámica sobre los diversos problemas que hoy afligen a nuestra sociedad, podemos ver que estos convergen en una crisis multidimensional de la vida tanto social como natural (Prieur, 2020). Varios de los espacios de la vida cotidiana de la humanidad, así como de la propia dinámica y flujos materiales y energéticos de la naturaleza, hoy se ven alcanzados por la égida valorizante del capitalismo contemporáneo, por lo tanto, la naturaleza y la sociedad -como unidad histórico concreta - han sido sumergidos en una dinámica acorde a la explotación de la fuerza de trabajo para la apropiación capitalista del plusvalor producido por la clase obrera, la acumulación de capital, la caída tendencial de la tasa de ganancia y el despojo tanto de los medios de producción como de subsistencia sociales que marcan la impronta de la especificidad del capitalismo contemporáneo (Lastra, 2020).

Nuestro tiempo se ha caracterizado, por un lado, por la producción de un desperdicio generalizado (Mészaros, 2007) y como en ambos casos una destructividad del capital globalizante (Mészaros, 2010). Desde la segunda mitad del siglo XX y las dos décadas de lo que va del XXI, el desarrollo histórico del modo de producción capitalista se ha caracterizado por la complicación de la subsunción real del proceso de trabajo bajo el capital (Marx, 1861 ) hasta devenir en una subsunción real del consumo bajo el capital (Veraza, 2009). De forma tal que la principal política de nuestra sociedad ha sido la producción de valores de uso nocivos y degradantes tanto para la vida humana como para las condiciones bio-químicas y geofísicas del planeta, en tanto que ha impulsado el desarrollo de cierto tipo de fuerzas destructivas (Barreda, 20 19) nucleadas por una tecnología del capital socioambientalmente nociva (Veraza, 2012). Este contexto histórico estructural ha terminado por acelerar las tasas de extracción de materiales y agua del territorio mexicano, con consecuencias medioambientales asoladoras que provocan la multiplicación de conflictos socioambientales (Tetreault, 2020).

En el marco de la referida crisis civilizatoria generada en pleno auge del desarrollo capitalista, uno de los productos históricos de nuestro tiempo es la devastación ambiental que hoy día se ha generado a escala planetaria (Arizmendi, 2005). Esta crisis energética, instrumental, material y simbólica de la naturaleza, se puede considerar como multidimensional, en tanto que, por ejemplo, se expresa en una crisis climática, un cambio en la integridad biosférica, un agotamiento de la capa de ozono, la acidificación oceánica, en alteraciones del ciclo biogeoquímico del nitrógeno y fósforo, el cambio de uso de suelo, la alteración del ciclo del agua, la carga atmosférica de aerosoles y la introducción de contaminantes novedosos de alta toxicidad (Rockström et al., 2009; Steffer et al., 2015).

El contexto ambiental actual se ha complejizado durante el neoliberalismo hasta el punto de ser considerado como 
una desfiguración retorcida del principio esperanza de la humanidad (Barreda et al., 2019). Esta afirmación, se debe de entender contraria a la noción eternizante e inespecífica con la que Simmel (2011) da cuenta de la conflictividad como dimensión caracterizante de la vida social contemporánea. Al referirnos al conflicto lo hacemos como una expresión directa de la contradicción valor de uso/valor presentada por Marx como parte nodal de su discurso crítico comunista (Echeverría, 1986) que tanto él como Engels Ilevan a cabo para desplegar una crítica global a la sociedad burguesa.

Bajo este contexto, se puede reconocer cómo, en el neoliberalismo, la tecnología capitalista nociva y el desarrollo de fuerzas productivas ambientalmente destructivas se sustentan en un marco epistemológico y tecnocientífico que han terminado por generar severas externalidades socioambientales que reactualizan y complican los históricos problemas sociales relacionados con la escasez material y producción de una miseria generalizada como parte de la expresión de la ley general de la acumulación de capital (Marx, 1867). Un ejemplo característico de lo recién mencionado, lo podemos encontrar en los conflictos socioambientales que, en las últimas tres décadas, han tupido los territorios, los cuales ya no se relacionan sola y exclusivamente con el acceso a tal o cual recurso natural o nivel de riqueza material, en tanto que no sólo se llevan a cabo conflictos de distribución objetiva de la naturaleza (Martínez-Allier, 2004), sino que, durante el neoliberalismo, la conflictividad socioambiental se encuentra encadenada a diversas actividades legales, ilegales o criminales de sobreexplotación, contaminación de la naturaleza y violación de los derechos humanos y ambientales tanto individuales como colectivos de las comunidades que han tenido que enfrentar el actuar colosal de los megaproyectos y megainfraestructuras productivas, urbanas, energéticas, extractivistas, comunicacionales y de transporte que hoy en día caracterizan la reconfiguración capitalista de los territorios.

El despojo de los medios de producción y de los medios de subsistencia ha marcado la impronta de la conflictividad socioambiental durante el neoliberalismo, sobretodo, a partir de diversas actividades extractivistas que han permeado a los territorios del planeta para intensificar el saqueo, sobreexplotación y contaminación de aquellos recursos naturales que resultan estratégicos para la reproducción y desarrollo del capital (Barreda, 2005). Como sugiere Tetreault (2020), la expansión del extractivismo genera consecuencias devastadoras en el ambiente y resulta en una gran cantidad de comunidades directamente afectadas por dichos procesos. Lo cual produce ciertas condiciones objetivas para provocar la eventual organización social para la defensa de los territorios, de los bienes naturales y de las dimensiones procreativas que estos tengan para la población.

La dinámica creciente del extractivismo a nivel mundial ha terminado por ser un factor que apunta hacia la superación de las fronteras planetarias (Rockström et al., 20091, y que, por lo tanto, se torna en una condición para la fractura metabólica entre la sociedad y la naturaleza (Padovan, 2015). El concepto de metabolismo, tal y como lo propone originalmente Marx dentro de las ciencias sociales, permite reconocer 1) la dialéctica fundacional entre el ser humano y la naturaleza; 2l el grado de correspondencia de las múltiples concatenaciones presentes en los procesos de transnaturalización de lo específicamente 
social y la socialización político-procreativo de lo propiamente natural. Por tal motivo, los conflictos socioambientales pueden ser pensados - aunque no de forma exclusivaa partir de los diversos procesos de extracción, distribución y consumo productivo-social de las energías, materiales y biomasa que hoy día resultan estratégicos para la acumulación de capital.

A partir de lo anterior, el presente estudio se plantea como propósitos específicos: 1) proponer una caracterización de los conflictos socioambientales contemporáneos a partir de su especificidad histórico concreta, considerándolos como un producto reactualizado por el neoliberalismo; 2) presentar una definición de los conflictos socioambientales desde la perspectiva ontoepistemológica y conceptual de la crítica de la economía política y el materialismo histórico; 3) abordar los niveles de extracción de los recursos naturales estratégicos que forman parte nodal del metabolismo social-natural en México, cuya tasa de explotación se establece como condición de posibilidad necesaria - más no explicativa de forma unidimensional - para la eventual emergencia de conflictos socioambientales.

\section{METODOLOGÍA}

\section{TIPO DE ESTUDIO}

De acuerdo con el propósito establecido, se realizó un análisis documental de corte cualitativo (Rodríguez y Luna-Nemecio, 2019) con el fin de sintetizar e interpretar las concepciones existentes sobre conflictos socioambientales, analizarlas de manera crítica y proponer una conceptualización y lectura basada en su especificidad histórica, así como en los elementos conceptuales y ontoepistemológicos de la crítica de la economía política (Marx,
1867). La investigación se hizo mediante la búsqueda de artículos y libros que ayudaran a tener claridad de los vacíos en torno a la conceptualización de este proceso y que brindasen orientaciones para un abordaje científico de los conflictos socioambientales en México en referencia al extractivismo, el metabolismo social y los recursos naturales estratégicos.

No se hizo una revisión exhaustiva de la literatura publicada sobre la conflictividad socioambiental; se buscaron artículos que permitieran mejorar la conceptualización y categorización de este término desde la crítica de la economía política, debido a la importancia social y académica de abordar dicha temática. Las cifras sobre las tasas de extracción de los recursos naturales estratégicos para el desarrollo del capitalismo nacional y transnacional en México son tomadas de Tetreault (2020), por lo que la originalidad de dicha información corresponde exclusivamente a él. Además, se retoman algunos elementos de su argumento sobre la relación entre las crecientes tasas de extracción de materiales y agua de México y la multiplicación de conflictos socioambientales. En este sentido, se propone que la aceleración del metabolismo social y las tecnologías empleadas establecen como condición de posibilidad necesaria - más no suficientepara la eventual emergencia de conflictos socioambientales, en tanto que representan factores que impactan negativamente en el ambiente y el tejido social de las comunidades rurales directamente afectadas. En el presente estudio se parte de esta información para relacionarla con datos del Atlas de Justicia Ambiental (EJATLAS, 2020) sobre conflictos socioambientales en México, enfatizando en los límites ecológicos planetarios que se ven tocados por dichas actividades extractivistas. 


\subsection{CATEGORÍAS DE ANÁLISIS}

A partir del propósito del estudio y de la analizó la información extraída de los revisión de la literatura, se plantearon las artículos encontrados en las bases de datos, categorías de análisis que se describen en aplicando un proceso de interpretación de la Tabla 1. Con base en ellas se organizó y corte hermenéutico.

\begin{tabular}{ll}
\hline Categoría & Preguntas para el análisis \\
\hline Conflictos socioambientales & ¿Cuál es la especificidad histórica concreta de los \\
& conflictos socioambientales? ¿Cómo se pueden \\
& conceptualizar y caracterizar los conflictos \\
& socioambientales desde la crítica de la economía \\
& política? \\
Extractivismo, metabolismo social & ¿Cuáles son las tasas de extracción de los recursos \\
y recursos naturales estratégicos & naturales estratégicos en México? ¿Qué relación guarda \\
& la conflictividad socioambiental con el saqueo, \\
& sobreexplotación y contaminación de aquellos recursos \\
& naturales que resultan estratégicos para la explotación \\
& de plusvalor y la acumulación de capital? \\
\hline
\end{tabular}

Tabla 1. Categorías de análisis de la investigación. Fuente: Elaboración propia

\subsection{CRITERIOS DE BÚSQUEDA DE DOCUMENTOS}

De acuerdo con el propósito de la investigación, se buscaron artículos y libros académicos considerando las categorías planteadas para el estudio conceptual, con base en la siguiente palabra clave "conflictos socioambientales" y su cruce con otras tales como "definición", "características", "diferencias", "crítica de la economía política", "devastación ambiental" "neoliberalismo", "extractivismo". Sólo se buscaron los documentos más pertinentes para abordar las categorías seleccionadas.

\section{RESULTADOS}

\section{ESPECIFICIDAD HISTÓRICA DE LOS CONFLICTOS SOCIOAMBIENTALES}

La emergencia de la conflictividad socioambiental en el marco de la devastación ambiental contemporánea en su especificidad neoliberal, se da precisamente en un contexto de sobreexplotación creciente de la naturaleza, de sobreconsumo de materiales y energía y de excreta inconmensurable de residuos sólidos y de alta toxicidad que hoy día confluyen, metabolizan, sincronizan y reaccionan químicamente para generar una contaminación ambiental nunca antes vista, es decir, que la emergencia actual de conflictos socioambientales toma el talante de una novedad histórica al estar inmersos en una devastación ambiental generada por la subsunción real del consumo por el capital específicamente neoliberal, en tanto que se ha acelerado vertiginosamente hacia un dislocamiento de los ciclos metabólicos de la naturaleza, motivado por el incremento en la capacidad histórica territorial del capital para destruir el ambiente, mediante el saqueo y contaminación inmensa e imparable de la naturaleza.

La devastación ambiental específicamente neoliberal no sólo tiene que ser entendida 
como un contexto general en el que emergen una cantidad mayor de conflictos socioambientales cuya estrategia de lucha y composición se torna cada vez más compleja, dados los escenarios de saqueo, contaminación y sobreexplotación del territorio, así como también por la emergencia de nuevos actores sociales responsables de la referida devastación. Por ejemplo, los cárteles de la droga que han impulsado directa e indirectamente procesos de despojo, privatización y violencia generalizada como parte de una ingeniería de conflicto que facilite la promoción de megaproyectos (Gasparello, 2017). Además, la referida devastación ambiental se debe entender como la condición de posibilidad sine qua non para la movilización de comunidades en contra del capital privado o del capital social, en tanto que es, precisamente, esta forma general del capital la que ha tornado inviable la vida en los territorios en disputa (Luna-Nemecio, 2019).

Es importante, entonces, que se proponga una forma de pensar la conflictividad socioambiental que permita ir en contra de la destotalización histórica y desespecificación del presente (Barreda, 2019). Por este motivo, se ha de considerar que los conflictos socioambientales no pueden ser abordados sola y exclusivamente desde su caracterización como un producto específicamente capitalista, sino que se tiene que abordar según su especificidad histórico concreta, a saber: deben ser considerados como un resultado del carácter caprichoso, deliberado, doloso, irresponsable, sádico y francamente criminal que ha tomado la economía y la política en el contexto histórico epocal del capitalismo específicamente neoliberal (Barreda et al., 20191.
Bajo dicho contexto cabe preguntarse jla conflictividad socioambiental puede pensarse como una territorialidad degradada y que, por lo tanto, en tanto valor de uso nocivo, corresponda a la subsunción real del consumo bajo el capital? (Veraza, 2009). La respuesta, según la perspectiva ontoepistemológica de la que parte esta investigación, es afirmativa en tanto que se considera a los conflictos socioambientales como un fenómeno socioterritorial que ocurre como condición y resultado de una acumulación originaria, residual y terminal de capital y del predominio de cierto tipo de tecnología capitalista nociva que emana del desarrollo de fuerzas productivas ambientalmente destructivas (Veraza, 2007).

En este tenor es importante que los conflictos socioambientales sean pensados a la luz de la reconfiguración neoliberal de la producción del espacio social (Orueta \& Seoane, 2013) y de la ley de población que se deriva de la ley general de la acumulación de capital expuesta por Marx en el capítulo XXIII del tomo I de "El capital" (Marx, 2017). El estudio sobre los conflictos socioambientales debe dar cuenta de los procesos sociales de lucha y resistencia popular ante los procesos de urbanización, industrialización, proletarización y violencia generalizada en el marco del neoliberalismo, en tanto que la dimensión humana de la conflictividad socioambiental representa un ejercicio de expresión de la politicidad básica de los sujetos (Echeverría, 1996) que se confronta con el desvío de poder político llevado a cabo por el Estado nacional durante el neoliberalismo para que, violentando la ley o con la ley en mano, legitimice el proceso de entrega de los territorios y de los recursos naturales a terratenientes y empresarios tanto locales como transnacionales (Espinoza, 20011. Además, se tiene que considerar 
que la generación de injusticia ambiental se da tanto por el actuar de empresas privadas en contubernio con el Estado mexicano, así como por medio de la acción de grupos criminales ligados al narcotráfico.

Para comprender la configuración de escenarios de conflictividad socioambiental, es importante dar cuenta del riesgo social que durante el neoliberalismo ha representado la imprevisibilidad que caracteriza los procesos de producción del espacio urbano y el apuntalamiento de conformación de corredores industriales emplazados sobre el territorio por parte del capital privado y social, o por aquellos actores que pertenecen a la economía criminal (Giraldo, 2013). Lo cual ha representado una ruptura tanto en el metabolismo socioambiental como en el propio carácter comunitario y restitutivo de toda la praxis humana. Por tales motivos, para que los conflictos socioambientales emergieron con tal efervescencia en diversos territorios del planeta durante el neoliberalismo, el riesgo producido por el capital tienen que impactar de forma efectiva sobre la naturaleza a un grado tal de que dicha afectación sea considerada como relevante o grave y que, de forma inminente, signifique la producción de condiciones ambientales y sociales degradadas sobre aquellos bienes naturales que las comunidades afectadas consideren y asuman como propios (Barreda y García-Barrios, s.f).

Para la emergencia de conflictos socioambientales se deben considerar la producción de ciertas condiciones objetivas y subjetivas de posibilidad. Dentro de las primeras se encuentra la escasez que se ha generado de forma natural 0 se ha producido socialmente y que ha terminado en la creación de impactos latentes, posibles o consumados sobre los bienes naturales. Además, dentro de las condiciones de corte subjetivo presentes en las comunidades para la emergencia de conflictos socioambientales, están: 1) la relación de identidad simbólica y práctico cultural que, en términos procreativos, sus integrantes tengan con la naturaleza; y 21 el predominio del sentido de lo colectivo-comunitario por encima del avance de la privatización de los medios de producción y de subsistencia (Barreda y García-Barrios, s.f).

\section{ECONOMÍA POLITICA DE LOS CONFLICTOS SOCIOAMBIENTALES}

Bajo la lógica de la crítica de la economía política, la emergencia de conflictos socioambientales está en la agudización creciente de la devastación ambiental, la degradación del tejido social y la producción social del riesgo y vulnerabilidad múltiple de las condiciones práctico materiales y simbólicas identitarias de la vida de las comunidades agraviadas. Los afectados ambientales toman actitudes de resiliencia (Altieri, 2013) para sacar adelante estrategias de organización, lucha y resistencia en contra de aquellos actores responsables de la referida devastación, teniendo como objetivo la restitución de sus derechos humanos y ambientales violentados.

La conflictividad socioambiental se puede considerar como un resultado de las actuales tendencias de la industrialización y urbanización insostenible del territorio (Luna-Nemecio, 2017); así como por la reconfiguración que el capitalismo neoliberal hace de éste para apuntalar sus 
procesos de reproducción y desarrollo (Tetreault et al., 2019). La conflictividad socioambiental puede ser considerada como el momento en que se patentiza prácticas de irresponsabilidad y/o incapacidad del capital industrial - tanto privado como social - para hacer frente, ya sea técnica o financieramente, a la serie de externalidades ambientales (Reynoso y Montes, 2016l que se generan como correlato a la producción de mercancías; siendo la sociedad en su conjunto quien ha de sufrir los efectos ecológicos de dicha devastación ambiental. Según Quintana (s.f), la conflictividad socioambiental se traduce en una serie de protestas sociales que buscan visibilizar e incluso frenar el avance de la devastación ecológica por parte de las industrias o ciudades.

Los conflictos socioambientales emergidos en el seno del neoliberalismo, desde una lectura acorde al discurso crítico de Marx, pueden ser esbozados como formas problemáticas y contradictorias de las relaciones sociales basadas en escenarios de pugna y confrontación referentes a la apropiación, saqueo y contaminación de los recursos naturales y de los territorios que terminan por poner en riesgo las condiciones biofísicas y geoquímicas de los recursos naturales que son estratégicos para la reproducción social. Los conflictos socioambientales son un resultado inmediato de la devastación ambiental ocasionada por la reconfiguración capitalista del territorio bajo en un contexto de generación de corredores urbano-industriales por los que fluyen energías, materiales, mercancías y fuerza de trabajo, bajo un contexto de desarrollo de tecnología capitalista nociva y fuerzas productivas ambientalmente destructivas.
La devastación socioambiental que ha terminado por caracterizar nuestro tiempo presentándonos como una normalidad degradada y degradante, participa de forma decisiva en la producción social de los territorios, generando diversos escenarios de riesgo y vulnerabilidad multidimensional de la totalidad de las condiciones de vida de las personas; implicando la generación de condiciones de posibilidad para la emergencia de movilizaciones sociales para denunciar la serie de agravios e injusticias socioambientales, ya sea para fincar responsabilidades o para exigir la reparación del daño (Espinosa, 2015). De alli que la conflictividad socioambiental se consideren espacios de praxis política tanto por el papel que juega el desvío de poder por parte del Estado y sus instituciones gubernamentales, así como por el propio despliegue de la politicidad básica de los sujetos que integran las comunidades y colectivos que participan activamente en la defensa de la naturaleza y sus territorios.

De forma tal que la conflictividad socioambiental surge en un contexto de escasez material, ya sea natural, pero, sobre todo, producida artificialmente por el capitalismo dada la lógica y estructura que toman las fuerzas productivas, la acumulación de capital y los procesos de subsunción formal y real del proceso de trabajo y del consumo bajo el capital; situación de escasez que está materialmente arraigada y objetivamente practicada en la vida cotidiana $y$ en el marco de necesidades sociales individuales y colectivas. En este sentido, los conflictos socioambientales deben ser entendidos como expresión directa de la búsqueda colectiva por el rescate y reivindicación de los derechos ambientales y sociales de la 
humanidad; son espacios donde el valor de uso busca empoderar para enfrentar y resistir ante el avance crematístico del valor como forma social hegemonizante.

Tal y como advierte Espinosa (2015), es importante comprender que en la conflictividad socioambiental que emerge del despliegue territorial de fuerzas productivas ambientalmente destructivas, se lleva a cabo una importante base social que desempeña un papel toral en los procesos de lucha y desarrollo de los mecanismos populares de lucha y resistencia colectiva frente a la creciente devastación ambiental de los territorios. Los conflictos socioambientales se tornan territorios de emancipación posibles y de un principio esperanza para la humanidad, en tanto que se acompañan con un claro despliegue de la politicidad básica de los sujetos que convierten a las comunidades y sus integrantes en un nuevo sujeto histórico capaz de llevar a cabo la reconquista de los derechos ambientales de aquellas personas y territorios afectados y que durante el neoliberalismo han sido vilipendiados más de una vez por unos y por otros.

\section{DINÁMICAS EXTRACTIVISTAS DE LOS RECURSOS NATURALES ESTRATÉGICOS EN MÉXICO, FRACTURA METABÓLICA Y CONFLICTIVIDAD SOCIOAMBIENTAL}

La devastación socioambiental de México por las dinámicas de reconfiguración urbana e industrial del territorio y por las actividades extractivistas que le acompañan, ha generado la producción masiva de afectados ambientales a lo largo y ancho del territorio nacional. Durante el gobierno de Enrique Peña Nieto se contabilizaron más de 514 sitios contaminados (Albert y Jacott, 2015) y se identificaron 124 emergencias ambientales (Blancas,
2014). Estos problemas ambientales nacionales, se han traducido en la conformación de conflictos socioambientales cuya clasificación puede ser de tipo agrícola, biotecnológico, energético, forestal, hidráulicos, mineros, por residuos tóxicos y rellenos sanitarios, así como relacionados con actividades turísticas y de desarrollo urbano o industrial (Espinoza et al., 2019; Toledo, 20151

Según datos de Tetreault (2020), en México la extracción de recursos naturales estratégicos (minerales, combustibles fósiles, biomasa y agual se ha incrementado aceleradamente entre 1990 y 2018, lo cual 1) evidencia una dinámica en los procesos extractivistas que se desarrollaron en el territorio mexicano durante el neoliberalismo y que terminan por incidir en la configuración tendencial de una fractura metabólica al contribuir a la superación de los límites o fronteras ecológicas del planeta; y 21 explica-aunque no de manera general ni suficiente- las condiciones materiales y biofísicas que han nutrido la multiplicación de los conflictos socioambientales en defensa del territorio a lo largo del territorio mexicano.

En lo que respecta a las actividades que la megaminería metálica ha desarrollado en México, se puede observar niveles de extracción de casi 300 millones de toneladas de materiales en 2018 (Tetreault, 2020). Esto ha significado que se avance hacia un cambio en el uso de suelo, la pérdida de la integridad biosférica y la creciente y cada vez más compleja introducción de elementos químicos novedosos de alta toxicidad que se emplean en la minería a cielo abierto. Por lo que el extractivismo de minerales metálicos y no metálicos se torna en un factor que presiona hacia la superación de las fronteras ecológicas que delimitan el frágil equilibrio 
metabólico entre la sociedad y la naturaleza. En este contexto, en México se contabilizan 37 conflictos socioambientales relacionados con megaproyectos mineros (EJATLAS, 2020).

La extracción y distribución de combustibles fósiles (gas y petróleo) en México, el estudio de Tetreault (2020), menciona que las dinámicas extractivas de hidrocarburos alcanzaron los 240 millones de toneladas en 2006, para posteriormente declinar hasta 142 millones de toneladas hacia fines de 2018, debido al agotamiento de las principales reservas del país. Dicha dinámica contribuye, por un lado, a la alteración en el ciclo biogeoquímico del nitrógeno, al agotamiento de la capa de ozono y a la generación intensiva de gases de efecto invernadero que agudizan la crisis climática global; volviéndose en elementos que apuntalan el ya de por sí vertiginoso arribo hacia una fractura metabólica global. Por otro lado, la extracción de hidrocarburos en México, ha contribuido a la emergencia de 18 conflictos socioambientales relacionados con los combustibles fósiles y la justicia energética/climática que para el año de 2020 continúan en un estado de vigencia y algidez (EJATLAS, 2020).

El consumo productivo y distributivo de biomasa extraída que generan los cultivos primarios, forrajeros, de pastizal, madereros en México alcanzó un total de 360 millones de toneladas para el año 2018 (Tetreault, 2020). Lo cual se ha traducido en 14 conflictos socioambientales relacionados con la extracción de biomasa y la defensa de los bienes forestales, la agricultura, pesca y ganadería (EJATLAS, 20201, pues las comunidades de diversas partes de México han tenido que luchar y resistir en contra de procesos productivos que impactan nodalmente en el metabolismo entre la sociedad y la naturaleza al generar una aceleración de la superación de las fronteras ecológicas planetarias en lo que tiene que ver con la integridad biosférica, el equilibrio entre los determinantes del ciclo del fósforo y el tipo de uso de suelo, por tan sólo mencionar algunos de los impactos que el extractivismo de biomasa genera sobre el planeta.

El agua, en tanto recurso hídrico estratégico para el desarrollo capitalista (Luna-Nemecio, 20181 , se ha vuelto un valor de uso altamente extraído mediante procesos hidroútiles que, durante el neoliberalismo, tomaron la forma de megaproyectos extractivistas. Para el caso mexicano, los niveles de extracción del vital líquido corresponden a 87.850 millones de toneladas para el año 2017, con base en las concesiones de Conagua (Tetreault, 2020). Esta situación se acompaña con la configuración de 22 conflictos socioambientales relacionados con el agua (EJATLAS, 2020) que han vuelto evidente el papel del extractivismo hídrico en la superación de los límites planetarios correspondientes tanto a la acidificación y contaminación residual y toxicológica de los recursos hídricos, así como a la alteración urbana e industrial del metabolismo hídrico.

\section{DISCUSIÓN}

Durante el neoliberalismo, los conflictos socioambientales han tomado la particularidad de estar centrados en el impacto posible, latente inminente o consumado sobre el ambiente. La emergencia de la conflictividad por la defensa de la naturaleza reconoce que el impacto sobre ésta lacera no sólo el consumo productivo que empresas privadas - el Estado nacional lleva a cabo para apuntalar procesos de explotación de plusvalor y acumulación de capital, sino 
que también toma como base la dimensión reproductiva que la naturaleza saqueada, explotada y contaminada representa para las comunidades que tienen una relación procreativa con dichos bienes naturales. De forma tal que los conflictos socioambientales en defensa del agua pasan por reconocer el talante de este vital líquido como un recurso natural estratégico, y por enfrentar un escenario de riesgo y vulnerabilidad múltiple, en tanto que la devastación de la naturaleza avanza hacia la fractura del metabolismo sociedad-naturaleza en su totalidad.

La caracterización de los conflictos socioambientales desde el discurso crítico de Marx esbozada líneas arriba, establece una diferenciación directa con la forma cómo la economía ecológica les observa, en tanto que ésta última les considera como conflictos ecológico-distributivos (Martínez-Alier, 2004) que no son más que una interpretación de la conflictividad socioambiental desde la perspectiva de un imperialismo ambiental. Este mirador se posiciona como crítico a las interpretaciones de corte marxista respecto a la conflictividad socioambiental en tanto que prohíbe el uso del concepto de lucha de clases para dar cuenta de la expresión de la economía política de los conflictos.

En este sentido, es importante reconocer algunas de las clasificaciones con la que son abordados los conflictos socioambientales. Por ejemplo, Paz (2017) considera distintas formas de cómo ha sido abordada la conflictividad socioambiental. En primer lugar, se encuentran los conflictos socioambientales motivados por dimensiones institucionales y en referencia a los espacios para la toma de decisiones; bajo esta clasificación autores como Vega (2014) y Brenner (2010) explican la conflictividad en referencia a una serie de desacuerdos entre los actores e intereses que pueden ser objetos de negociación y normatividad. Sin embargo, dichas interpretaciones, aunque reconocen la importancia de la habilidad política para lograr una negociación y la toma de acuerdos, no dan cuenta del papel que juegan el poder como un factor que rompe con la supuesta relación entre iguales que intenta argumentar esta perspectiva.

En segundo lugar, se encuentran los conflictos ecológico distributivos presentados por estudios como los elaborados por Martínez-Alier (2004) y Walter (2009). Estos autores marcan como dimensión central de la emergencia de la conflictividad socioambiental la presencia de condiciones económicas y políticas de una distribución desigual de beneficios y de los riesgos y vulnerabilidades derivados de la sobreexplotación y contaminación de la naturaleza. Esta forma de concebir a los conflictos socioambientales tiene de correcto ubicar la distribución de los efectos ambientales en los países periféricos como parte de una explicación de la distribución geográfica de la conflictividad socioambiental a partir de la teoría dependentista y de las teorías del imperialismo (Veraza, 2002).

Sin embargo, esta interpretación tiene el límite de no reconocer que, conforme el capitalismo adopta una medida geopolítica mundial (Veraza, 2010) y va desarrollando sus fuerzas productivas técnicas, procreativas y generales, logra que los procesos productivos no sólo se instalen en aquellas zonas de la periferia donde existía una mayor abundancia de recursos naturales estratégicos y de población, sino que, ahora, conforme se va estructurando una red global de corredores urbano-industriales que tienden a conformar un sistema automático de máquinas (Barreda, 2006), el saqueo, sobreexplotación y contaminación de los recursos naturales no ocurre sola y 
exclusivamente en la referida periferia, sino que es en el núcleo de la metrópoli misma donde ocurren también afectaciones ambientales sin importar el nivel de ingreso e, incluso, sin tomar en cuenta el carácter de clase social. Esta afirmación tiene que ser matizada, pues si bien hay saqueo y destrucción ecológica en los países centrales, lo cierto es que -tanto en el Norte como en el Sur- la injusticia ecológica sigue siendo una dimensión importante de la cuestión ambiental en los territorios que coronan el desarrollo territorial de los países en donde el capitalismo se ha consolidado. Es decir, los impactos socioambientales del extractivismo y la contaminación afectan de manera más directa y desproporcionadamente a las comunidades marginadas (urbanas y rurales) en aquellas economías emergentes o en vías del desarrollo de los mecanismos de subsunción formal y real del proceso de trabajo por el capital.

Paz (2017), menciona que se pueden clasificar los conflictos socioambientales como ambientalmente inducidos; según Walter (2009) este tipo de conflictividades son resultado de la escasez de recursos derivado de la devastación socioambiental. Sin embargo, al considerar la variable de la presión social sobre la naturaleza, se acercan a interpretaciones neomalthusianas sobre la devastación ambiental. Además, de reducir la conflictividad socioambiental a una dimensión distributiva y desvinculada a lo productivo, los autores que realizan investigaciones desde esta perspectiva carecen de un fundamento teórico y metodológico específico. Dentro de la conflictividad socioambiental recién aludida, se tiene que reconocer aquellos que tienen como centro la defensa de los recursos naturales estratégicos como lo es el agua, al buscar enfrentar la creciente contaminación, saqueo, despojo y privatización de los cuerpos superficiales y subterráneos del vital líquido, así como de los procesos de extracción, distribución y saneamiento de los recursos hídricos que ha representado el tipo particular de desarrollo urbano-industrial del territorio durante el neoliberalismo (Tetreault y McCulligh, 20181.

Los resultados del presente estudio argumentan la importancia de observar conflictos socioambientales desde el mirador iconoclasta del discurso crítico de Marx. Esta perspectiva ontoepistemológica guarda una serie de diferencias respecto a los discursos hegemónicos que han tomado como objeto de análisis y reflexión la categorización de este fenómeno social. Por ejemplo, la teoría de los Nuevos Movimientos Sociales (Santos, 2001) abordados desde una ontoepistemología postmaterialista (Pigem, 2010) presenta una caracterización de los conflictos socioambientales que resulta altamente criticable por 1) confundir el valor de uso de la naturaleza con la forma valor; 2) ofrecer una lectura sobre la conflictividad socioambiental sin reconocer la lucha de clases; 3) no hay una crítica sobre el tipo de fuerzas productivas ni de la tecnología capitalista que predomina en nuestro tiempo, lo cual crea una confusión entre el crecimiento económico y la sostenibilidad; 4) reactualiza las teorías del imperialismo para ofrecer una distinción entre la presencia/ausencia de nuevos valores ambientales ligados al desarrollo económico y social que se despliegan diferencialmente en los países del Norte y del Sur; 51 argumentar en términos neomalthusianos los factores de explicación de la devastación ambiental y, por lo tanto, de la conflictividad socioambiental; y 6) ofrecer una perspectiva postindustrial y transhumanista que presenta como inespecífica el papel activo del capitalismo gran industrial como uno de los principales actores en la devastación ambiental global. 


\section{CONCLUSIONES}

La lectura sobre los conflictos socioambientales desde la crítica de la economía política propuesta como resultado de este estudio, se diferencia de aquellas interpretaciones que buscan subrayar el papel del despojo o la mercantilización de la naturaleza como explicaciones unidimensionales de los conflictos socioambientales (Composto, 2012; Navarro, 2012). A contrapelo de estas interpretaciones, nuestra propuesta de lectura sobre este tipo de fenómenos pasa por reconocer que la emergencia y complicación de los conflictos socioambientales desarrollos durante el capitalismo específicamente neoliberal responde a la lógica y estructura de: 1) los procesos productivos centrados en la apropiación del plusvalor; 2) la acumulación cuantitativa y cualitativa de capital; 3) el desarrollo de fuerzas productivas técnicas y procreativas; 4) la contradicción valor/valor de uso; 5) la caída tendencial de la tasa de ganancia y las causas que le contrarrestan; y 5) el proceso general de subsunción formal y real del proceso de trabajo y del consumo bajo el capital.

Los resultados de investigación aquí presentados tienen la relevancia de 1) recuperar la investigación de frontera desarrollada por Barreda et al., (2019), Espinosa (2015) y Tetreault et al., (2019) para dar cuenta de una dimensión crítica de los conflictos socioambientales, a partir de recuperar la perspectiva ontoepistemológica y teórico conceptual del discurso crítico de Marx y de su crítica de la economía política. Partiendo de estos referentes, 2) se propone una lectura novedosa, fresca y actual sobre la economía y políitica de los conflictos socioambientales, destacando su especificidad como un producto específicamente neoliberal.

La conflictividad socioambiental -en tanto espacio de lucha de la población civil organizada contra la ambición y lógica crematística del capital global- 1) visibiliza los diversos movimientos sociales cuya agenda política va en contra de la construcción de megaproyectos, servicios ambientales, tala inmoderada de bosques, ampliación de carreteras y violación de los planes de ordenamiento ecológico territoriales; 21 evidencian el carácter insustentable que actualmente tiene la producción social del territorio a la luz del despliegue de proyectos de industrialización y urbanización que terminan por construir escenarios de riesgo y vulnerabilidad latentes y se caracterizan por al la dislocación metabólica de las condiciones biofísicas y geoquímicas de los límites ecológicos planetarios; b) las afectaciones de las relaciones sociales por la aplicación de una ingeniería de conflicto que termina por favorecer el desarrollo de un megaproyecto y debilitar la base política del movimiento social que le resiste o combate.

De tal forma, es poco conveniente que los estudios en torno a la conflictividad socioambiental se queden en simples abordajes descriptivos que presenten las "percepciones sociales" del riesgo; mucho menos, la creciente conflictividad socioambiental no debe ser relativizada a un simple problema de gestión ante el cual se pueda adaptar y ser resilientes. Resulta importante que se desarrollen estudios de contexto sobre la conflictividad socioambiental que tomen como eje el discurso crítico de Marx, en tanto que éste toma como marco de referencia la crítica de la propiedad privada desde una perspectiva comunitaria basada en condiciones materiales de existencia. Además, el pensamiento de Marx permite reconocer la lucha que al interior de los conflictos 
socioambientales se da por el valor de uso frente al predominio histórico concreto, durante el neoliberalismo, de una tecnología capitalista nociva que avanza hacia el sometimiento de la vida social y natural sobre la lógica crematística de la valorización de valor.

\section{AGRADECIMIENTOS}

Se reconoce y agradece al Consejo Nacional de Ciencia y Tecnología de México (CONACYT) por financiamiento de la presente investigación en el marco del Programa Becas Posdoctorales por México 2020; al Dr. Darcy Tetreault por los comentarios y señalamientos críticos que permitieron mejorar los argumentos y por compartir los resultados de su investigación sobre extractivismo conflictos socioambientales, los cuales han sido parte central del análisis presentado en este estudio.

\section{REFERENCIAS}

Albert, L., Jacott, M. (2015). México Tóxico: emergencias químicas. Siglo XXI.

Altieri, M.A. (2013). Construyendo resiliencia socio-ecológica en agroecosistemas: algunas consideraciones conceptuales y metodológicas. Agroecología y resiliencia socioecológica: adaptándose al cambio climático, 94-104. Recuperado de hitps://bit.ly/2UtvMbe

Arizmendi, L. (2005). La crisis ambiental mundializada en el siglo XXI y sus disyuntivas. Revista Mundo Siglo XXI, (3), 17-36. Recuperado de http:// hall.handle.net/10469/7390

Barreda, A. (2005), Geopolítica, recursos estratégicos y multinacionales. Madrid: Pueblos, revista de Información y Debate.
Recuperado de http://www.revistapueblos.org/old/spip.php?article3 11

Barreda, A. (2006). Impacto ambiental y social global de las megainfraestructuras de transporte. Ecología política, (31), 41-51. Recuperado de http://mww.jstor.org/stable/20743646

Barreda A. (2019). Anatomía de la decadencia de la relación capitalista entre la sociedad y la naturaleza. En: Barreda, A., Enríquez, L., Espinoza, R. (2020). Economía política de la devastación ambiental y conflictos socioambientales en México (pp. 23-1411. Ed. Itaca.

Barreda, A., Enríquez, L., Espinoza, R. (2019). Economía política de la devastación ambiental y conflictos socioambientales en México. Ed. Itaca.

Barreda, A., García-Barrios, R. (coords) (s.f). Afectación del sistema hídrico nacional por el Estado mexicano. Centro Regional de Investigaciones Multidisciplinarias/UNAM/Ed. Itaca.

Blancas, D. (2014). Van 1, 124 emergencias ambientales este sexenio. México: La Crónica Diaria. Recuperado de http://www.cronica.com.mx/notas/2014/854288.html

Brenner, L. (2010). Gobernanza ambiental, actores sociales y conflictos en las Áreas Naturales Protegidas mexicanas. Revista mexicana de sociología, 72(2), 283-310. Recuperado de http://www. s c i e l o. org. mx/s c i e lo.php?pid=SO 1 88-25032010000200 $004 \&$ script=sci_arttext

Composto, C. (2012). Acumulación por despojo y neoextractivismo en América 
Latina. Una reflexión crítica acerca del Gasparello, G. (2017). Entre la montaña Estado y los movimientos socio-ambientales en el nuevo siglo. Astrolabio, (8), 323-352. Recuperado de https://revistas.unc.edu.ar/index.php/astrolabio/

Echeverría, B. (1986). El discurso crítico de Marx. Ed. Era.

Echeverría, B. (1996). Lo político en la política. Theoría. Revista del Colegio de Filosofía, (4), 11-21. https://doi.org/10.22201/ffyl. 16656415 p. 1 997.4 .147

EJATLAS (2020). Conflictos socioambientales en México. Recuperado de https://ejatlas.org/country/mexico

Espinosa Hernández, R. (2015). Socio-environmental Conflicts and Poverty: The Case of the Metropolitan Area of Mexico City. Cuadernos de Geografía: Revista Colombiana de Geografía, 24(1), 193-201. http://dx.doi.org/10.15446/rcdg.v24n 1.41971

Espinoza, R. (2019). Despropósitos normativos y estrategias jurídicas para la reivindicación colectiva de derechos. En: Barreda, A., Enríquez, L., Espinoza, R. (2019). Economía política de la devastación ambiental y conflictos socioambientales en México (pp. 231-319). Ed. Itaca.

Espinoza, R., Rosas-Landa, O., Maza, A. Gómez, O., Martínez, A., Andrade, G. (2019). Los conflictos socioambientales de México (2011-2015). En: Barreda, A., Enríquez, L., Espinoza, R. (2019). Economía política de la devastación ambiental y conflictos socioambientales en México (pp. 179-230). Ed. Itaca. y Wirikuta. Defensa del territorio y del patrimonio cultural y natural de los pueblos indígenas. Argumentos: Estudios Críticos de la Sociedad, 221-238. Recuperado de https://www.redalyc.org/pdf/595/59551330011.pdf

Giraldo, J. (Ed.). (2013). Economía criminal y poder político. Universidad Eafit.

Harvey, D. (2006). La acumulación por desposesión. En: Bueno, C., Pérez, M. Espacios globales (p. 21-52). Universidad Iberoamericana/ Plaza y Valdés.

Lastra, F. (2018). La superexplotación de la fuerza de trabajo y la especificidad del capitalismo latinoamericano: un aporte al debate. Izquierdas, (38), 257-278. http://dx.doi.org/10.4067/s0718-50 492018000100257

Luna-Nemecio, J. (2017). La Insustentabilidad Socioambiental de la Producción del Espacio Urbano en el Capitalismo Específicamente Neoliberal. Revista de Geografía ESPACIOS, 61111, 89-109. $\mathrm{h} \dagger+\mathrm{p} s \mathrm{~s}: \mathrm{l} /$ doi.org/10.25074/07197209.1 1.609 Luna-Nemecio, J. (2018) El papel estratégico del agua frente a los proyectos de mega-infraestructuras urbanas e industriales y la reconfiguración del territorio por el capital. Revista de Geografía ESPACIOS, 8(16), 1 1-38. htips://doi.org/10.25074/07197209.16.1110

Luna-Nemecio, J. (2019). Reconfiguración del territorio y movimientos sociales: territorios en disputa. Tlalli. Revista de Investigación en Geografía, 1121, 55-75. hitps://doi.org/10.22201/ffyl.26832275e.2019. 2.1085 
Martínez-Alier, J. (2004). Los conflictos ecológico-distributivos y los indicadores de sustentabilidad. Revibec: revista de la Red Iberoamericana de Economía Ecológica, 1, 21-30.

Marx, K. (1861). La tecnología del Paz, M.F., Risdell, N. (2014). Conflictos, capital: subsunción formal y subsunción conflictividades y movilizaciones real del proceso de trabajo al proceso de socioambientales en México: problemas valorización: extractos del manuscrito, 1867-7863. Itaca.

Marx, K. (1867). El Capital. Crítica de la economía política. Siglo XXI de España Editores.

Mészáros, I. (2007). El siglo XXI: ¿̇socialismo - barbarie? Monte Ávila Eds. Latinoamericana.

Mészaros, I. (2010). El desafío y la carga del tiempo histórico: el socialismo en el siglo XXI. LOM Ediciones.

Navarro, M.L. (2012). Las luchas socioambientales en México como una expresión del antagonismo entre lo común y el despojo múltiple. OSAL, 13(32), $150-70$.

Orueta, F.D., Seoane, M.L.L. (2013). Neoliberalismo, políticas urbanas y reconfiguración socio-espacial. Quid 16. Revista del Área de Estudios Urbanos, (3), 7-16. Recuperado de https://bit.ly/21CgU87

Padovan, D. (2015). Metabolic exchanges and practices of regulation: The assemblage of environment and society in early social sciences. Ecological Informatics, 26, 6-17. https://doi.org/10.1016/j.ecoinf.2014. 02.006
Paz, M.F. (2017). Luchas en defensa del territorio. Reflexiones desde los conflictos socio ambientales en México. Acta Sociológica, 73, 197-219. https://doi.org/10.1016/i.acso.2017.08.007 comunes, lecturas diversas. CRIM, Centro Regional de Investigaciones Multidisciplinarias.

Pigem, J. (2010). Buena crisis: hacia un mundo postmaterialista. Editorial Kairós.

Pérez-Vega, I. (2020). El "desarrollo sostenible" es un engaño, aseguró Joan Martínez Alier. Guadalajara (México): 44 Noticias. Recuperado de https://udgtv.com/noticias/desarrollo-sost enible-engano-joan-martinez-alier/

Prieur, J. (2020). Critical warning! Preventing the multidimensional apocalypse on planet Earth. Ecosystem services, 45, 101-161. https://doi.org/10.1016/i.ecoser.2020.101161

Quintana, A.P. (s.f). El conflicto socioambiental y estrategias de manejo. FUHEM. Recuperado de https://www.fuhem.es/media/cdv/file/biblioteca/Conflictos_socioecologicos/conflicto_socioambiental_e strategias\%20_manejo.pdf

Reynoso, L.H., Montes, A.L. (2016). Impuestos ambientales al Carbono en México y su progresividad: una revisión analítica. Economía Informa, 398, 23-39. https://doi.org/10.1016/i.ecin.2016. 04.003 
Rodríguez, O., Luna-Nemecio, J. Tetreault, D., McCulligh, C. (2018). (2019). Educación musical para el Water Grabbing via Institutionalised desarrollo sostenible: una revisión docu- Corruption in Zacatecas, Mexico. Water mental. Revista da abem. Associação Alternatives, 11131. 572-591. Recuperado brasileira de educação musical, 27/43), de https://bit.ly/3npvWNy 132-149.

Tetreault, D., McCulligh., Lucio, C. (eds.) Rockström, J., Steffen, W., Noone, K., (2019). Despojo, conflictos socioambiental Persson, Å., Chapin III, F.S., Lambin, E., es y alternativas en México. Universidad Lenton, T., Scheffer, M., Folke, C., Schell- Autónoma de Zacatecas/Miguel Ángel nhuber, H., Nykvist, B., de Wit, C., van Porrúa.

der Leeuw, S. Rodhe, H., Sörlin, S.,

Snyder, P., Costanza, R., Svedin, U., Tetreault, D. (29 de octubre de 2020). Falkenmark, M., Karlberg, L., Corell, R., Extractivismo y conflictos socioambientales Fabry, V., Hansen, J., Walker, B., Liver- en México [Ponencia]. Seminario sobre man, D., Richardson, K., Crutzen, P., Conflictos Socioambientales y Alternativas Foley, J. (2009). Planetary Boundaries: en México, Zacatecas, México.

Exploring the Safe Operating Space for Humanity. Ecology and Society, 14(2). Toledo, V. (2015). Ecocidio en México. La Recuperado de http://www.- batalla final es por la vida. Ed. Grijalbo. istor.org/stable/26268316

Ulloa, A. (2017). Dinámicas ambientales y Santos, B.D.S. (2001). Los nuevos mov- extractivas en el siglo XXl: ¿̇ es la época del imientos sociales. OSAL: Observatorio Antropoceno o del Capitaloceno en LatinoSocial de América Latina, (5), américa?. Desacatos, (54), 58-73. Recu177-188. Recuperado https://bit.ly/38ExzTi

de perado de https://bit.ly/2UtJWcG

Vega, Y.T.H. (2014). La consulta previa en Simmel, G. (2011). El conflicto de la la solución de conflictos socio-ambientales. cultura moderna. Ed. UNC.

Steffen, W., Richardson, K., Rockström, lo.oa?id=85131029004 J., Cornell, S.E., Fetzer, I., Bennett, E.M., Biggs, R., Carpenter, S.R., de Vries, W., Veraza, J. (2002). Crítica a cuatro de Wuit, C.A., Folke, C., Gerten, D., interpretaciones de la historia del siglo XX: Heinke, J., Mace, G.M., Persson, L.M., Giovanni Arrighi, Paul Johnson, Eric Ramanathan, V., Reyers, B., Sörlin, S. Hobsbawm y Antonio Negri. Polis: (2015). Planetary boundaries: Guiding Investigación y Análisis Sociopolítico y human development on a changing Psicosocial, 2(2), 7-40. Recuperado de planet. Science, 347(6223). http.- https://www.redalyc.org/pdf/726/726 doi.org/10.1126/science.1259855 02202.pdf 
Veraza, J. (2009). Subsunción real del consumo Walter, M. (2009). Conflictos ambientales, bajo el capital. Dominación fisiológica y socioambientales, ecológico distributivos, psicológica de la humanidad. Ed. Itaca.

Veraza, J. (2010). Crisis económica y crisis de contenido ambiental... Reflexionando sobre enfoques y definiciones. Boletín Ecos, de la forma neoliberal de civilización (o de https: / / bit.ly/2UF3lYh la subordinación real del consumo bajo el capital específicamente neoliberal). Zamora, M.E., Huerta, A.H., Maqueo, Argumentos, 23(63), 123-157. O.P., Badillo, G.B., Bernal, S.I. (2016). Recuperado de https://bit.ly/34koOdd Cambio global: el Antropoceno. CIENCIA ergo-sum, Revista Científica MultidisciplinarVeraza, J. (2012). Karl Marx y la técnica desde ia de Prospectiva, 2311), 67-75. Recuperala perspectiva de la vida. Para una teoría do de http://www.redalyc.org/articumarxista de las fuerzas productivas. Ed. Itaca. lo.oa?id=10444319008 